\title{
Protocol design and performance analysis for cognitive cooperative networks with multiple antennas
}

\author{
Yuzhen Huang, Qihui Wu*, Jinlong Wang and Yunpeng Cheng
}

\begin{abstract}
In this article, we deal with a novel access protocol design for cognitive cooperative networks with multiple antennas. According to the principles of cognitive radio, the secondary user (SU) can exploit the primary user (PU) burstiness to access the licensed spectrum in the proposed access protocol. To get more access opportunities to the licensed spectrum, the SU simultaneously relays the PU's packets and transmits its own packets based on the superposition coding. Furthermore, to concurrent transmission of the PU's packets and SU's packets based on the superposition coding such that they are received without the interference at the primary receiver and secondary receiver respectively, two weight vectors at the SU equipped with multiple antennas are designed based on zero-forcing algorithm. Specifically, from a networking perspective, we analyze the performance of the proposed access protocol in terms of the maximum stable throughput and the average end-to-end delay for both the PU and SU based on the principles of queueing theory. In addition, to protect the PU's performance and exhibit the advantage of adopting multiple antenna technology, we jointly optimize the parameter of superposition coding and the number of antennas, and define the maximum stable throughput cooperative gain compared to the non-cooperative access scheme. More importantly, the impact of imperfect channel state information (CSI) at the SU on the maximum stable throughput and the average end-to-end delay performance is evaluated in the simulations from a practical point of view. Analysis and simulation results demonstrate that the proposed access protocol achieves significant performance gains for both the PU and the SU, outperforms the existing cooperative access protocol based on the dirty-paper coding, and keeps robust against to the imperfect CSI.
\end{abstract}

Keywords: Cognitive cooperative networks, Queueing theory, Stable throughput, Delay analysis, Zero-forcing algorithm

\section{Introduction}

Cognitive radio has been proposed as a promising technology to solve the contradiction between the inefficient utilization of spectrum and the limitation of spectrum resources in recent years [1]. In cognitive radio networks, the secondary user (SU) is allowed to share the licensed spectrum when the performance of the primary user (PU) is not affected. Currently, the SU can access the licensed spectrum through three schemes: interweaved spectrum sharing, underlay spectrum sharing and overlay spectrum sharing [2]. In the interweaved spectrum sharing scheme,

*Correspondence: wqhtxdk@yahoo.cn

College of Communications Engineering, PLA University of Science and Technology, Nanjing, China the $\mathrm{SU}$ can access the licensed spectrum when the PU is detected to be absent, known as spectral hole. In the underlay spectrum sharing scheme, the $\mathrm{SU}$ is allowed to share the licensed spectrum if the interference from the SU causing to the PU is below a given threshold, i.e., interference temperature. In the overlay spectrum sharing scheme, the SU needs to obtain the PU's message, such as modulation and coding modes, to aid PU's transmission to get more access opportunities. In this article, we focus on evaluating the performance of an overlay spectrum sharing system from a networking perspective.

Recently, relaying transmission has been proposed as a way to improve the throughput and coverage of wireless communication systems [3]. Considering the advantage of relaying transmission, it has been broadly applied in

\section{是 Springer}

(c) 2013 Huang et al.; licensee Springer. This is an Open Access article distributed under the terms of the Creative Commons Attribution License (http://creativecommons.org/licenses/by/2.0), which permits unrestricted use, distribution, and reproduction in any medium, provided the original work is properly cited. 
cognitive radio networks. Most of the existing works on the combined topic focus on solving the problems in the physical layer [4-10], such as the enhancement of the sensing ability for the SU and the improvement of the outage and error performances for both the PU and SU. When delay-sensitive applications are considered, other performance metrics about the maximum stable throughput and the average end-to-end delay become critical. Nowadays, various access protocols for cognitive cooperative networks were proposed where the SU cooperatively relayed the PU's packets in exchange for more access opportunities [11-14]. Simeone et al. [11] analyzed the maximum stable throughput for both the primary system and the secondary system with and without relaying capability in the basic four nodes with single antenna configuration. Sadek et al. [12] investigated the stability and the delay of a cognitive multiple access relay channel, where the cognitive user acted as a cooperative node for relaying PU's packets without having its own packets. Krikidis et al. [13] proposed various protocol designs for a single antenna cognitive cooperative system with a cluster of SUs by using dirty-paper coding (DPC) and opportunistic relay selection. However, the system model considered in [13] was not practical which required the information to be perfectly exchanged with the inner of the cluster and rigorous synchronization for each user. In addition, there was the interference at the primary receiver (PR) and the secondary receiver (SR) in the proposed access protocol based on the complicated DPC [13], which resulted in the poor performances of PU and SU. Bao et al. [14] studied the performance of a single antenna cognitive cooperative system with coexisting multiple PUs and one SU in terms of the maximum stable throughput and the average end-to-end delay.

While these prior works have improved our understanding on the protocol design and performance analysis for cognitive cooperative systems, the key limitation of these works is that they all assume a single antenna system. With the fact that the multiple antenna technique will be adopted as one of the key enabling technologies for the next generation wireless communication systems, the importance of understanding the fundamental performance of cognitive multiple antenna networks becomes increasingly evident [15-18]. Therefore, we are interested in the scenario where a SU equipped with multiple antennas and making a comprehensive analysis of the proposed protocol design for cognitive cooperative networks in this article. To the best of our knowledge, the evaluation of the application of multiple antenna technique to cognitive cooperative networks has not been reported in the literature from a networking point of view. Hence, the goal of the work is to evaluate the stable throughput for PU and $\mathrm{SU}$ and the end-to-end delay performance for the PU by using the principle of queuing theory. To get more access opportunities, we make the $\mathrm{SU}$ as a relay for the $\mathrm{PU}$ and allow the SU to simultaneously transmit the PU's packets and its own packets based on the superposition coding in the cognitive cooperative networks. Furthermore, to avoid the interference to each other, two weight vectors are designed at the SU for the PR and the SR based on zero-forcing algorithm, respectively. More importantly, the impact of imperfect channel state information (CSI) on the performance of the proposed access protocol is also considered in the simulations. Results demonstrate that the designed protocol is robust against to the imperfect CSI and achieves significant performance gains for the PU and the SU, respectively.

The rest of this article is organized as follows. In Section 2, a system model is described. Section 3 designs the cognitive cooperative protocol and analyzes the maximum stable throughput of the proposed access protocol for both the PU and the SU, respectively. In Section 4, we define the cooperative gain of the proposed cooperative protocol and jointly optimize the parameter of the superposition coding and the number of antennas. In Section 5, the average end-to-end delay performance for the PU is analyzed. Finally, conclusions are drawn in Section 6.

\section{System model}

As illustrated in Figure 1, we consider a cognitive cooperative system which consists of a PU, a PR, a SU equipped with $M$ antennas and a SR. ${ }^{a}$ We make an assumption that both the PU and SU have an infinite buffer to store the incoming packets as in [11-14]. The channel is slotted in time and the transmission time of each packet equals to a slot duration. The packets' arrival at the PU and the SU are Bernoulli random process, independently and stationary from slot to slot with mean $\lambda_{p}$ and $\lambda_{s}$ (packets per slot), respectively. Due to the effect of the fading channel, a packet can be successfully or unsuccessfully received by the intended receiver, which requires the feedback acknowledgment (ACK) and negative acknowledgment (NACK), ${ }^{b}$ respectively.

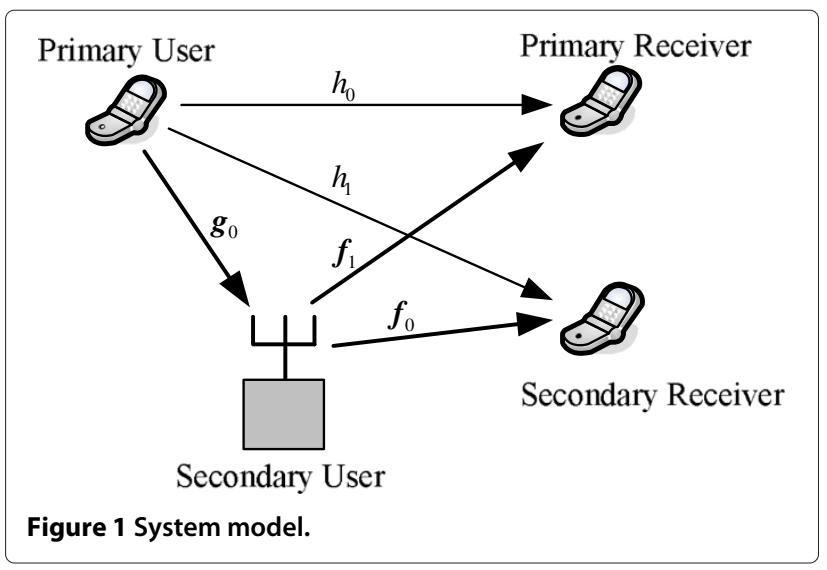




\subsection{Physical layer model}

We assume that all channels experience independent stationary Rayleigh flat-fading with channel coefficients being denoted as in Figure 1, which are circularly symmetric complex Gaussian random variable with zero-mean and unit-variance. Thus we have $h_{0} \sim \mathcal{C N}\left(0, d_{\mathrm{PU}, \mathrm{PR}}^{-\alpha}\right)$, $h_{1} \sim \mathcal{C N}\left(0, d_{\mathrm{PU}, \mathrm{SR}}^{-\alpha}\right), \mathbf{g}_{0} \sim \mathcal{C N}_{M, 1}\left(\mathbf{0}_{M}, d_{\mathrm{PU}, \mathrm{SU}}^{-\alpha} \mathbf{I}_{M}\right), \mathbf{f}_{0} \sim$ $\mathcal{C N}_{M, 1}\left(\mathbf{0}_{M}, d_{\mathrm{SU}, \mathrm{SR}}^{-\alpha} \mathbf{I}_{M}\right)$, and $\mathbf{f}_{1} \sim \mathcal{C N}_{M, 1}\left(\mathbf{0}_{M}, d_{\mathrm{SU}, \mathrm{PR}}^{-\alpha} \mathbf{I}_{M}\right)$, where $\alpha(2 \leq \alpha \leq 5)$ denotes the path-loss exponent and $d_{i j}, i \in\{\mathrm{PU}, \mathrm{SU}\}, j \in\{\mathrm{PR}, \mathrm{SR}\}$ represents the distance between the respective transmitter and receiver. Throughout the article, we assume that the CSI about $\mathbf{g}_{0}, \mathbf{f}_{0}$ and $\mathbf{f}_{1}$ is known at the SU. ${ }^{\mathrm{c}}$ The transmit powers at the PU and the SU are denoted by $P_{p}$ and $P_{s}$, respectively. $n_{k} \sim \mathcal{C N}\left(0, \sigma^{2}\right), \forall k \in\{\mathrm{PR}, \mathrm{SR}\}$ is complex additive white Gaussian noise (AWGN) at the PR and SR. $\mathbf{n}_{\mathrm{SU}} \sim$ $\mathcal{C N}_{M, 1}\left(\mathbf{0}_{M}, \sigma^{2} \mathbf{I}_{M}\right)$ is the AWGN vector at the SU. Accordingly, the signal $x_{p}$ received at the $\mathrm{SU}$ and the PR from the PU at time $t$ can be respectively expressed as

$$
\begin{aligned}
& \mathbf{y}_{\mathrm{SU}}=\sqrt{P_{p}} \mathbf{g}_{0} x_{p}+\mathbf{n}_{\mathrm{SU}} . \\
& y_{\mathrm{PR}}=\sqrt{P_{p}} h_{0} x_{p}+n_{\mathrm{PR}},
\end{aligned}
$$

where the index $t$ is dropped without loss of generality.

In this article, we consider that the success or failure of packet reception for each link $i \rightarrow j$ is characterized by outage events and outage probabilities. The outage event $O_{i j}$ is defined as the instantaneous achievable rate $C$ falls below a given rate $R$ with an outage probability $\operatorname{Pr}\left\{O_{i j}\right\}=\operatorname{Pr}\{C<R\}$. In order to overcome the impact of the fading channel and decrease the outage probability between the $\mathrm{PU}$ and the $\mathrm{SU}$, we apply a $1 \times M$ weight vector $\mathbf{w}_{r}$ to the received signal $\mathbf{y s U}_{\mathrm{SU}}$ at the $\mathrm{SU}$, then the resultant scalar signal at the $\mathrm{SU}$ is given by

$$
y_{\mathrm{SU}}=\sqrt{P_{p}} \mathbf{w}_{r}^{\dagger} \mathbf{g}_{0} x_{p}+\mathbf{w}_{r}^{\dagger} \mathbf{n}_{\mathrm{SU}},
$$

where $(\cdot)^{\dagger}$ denotes the conjugate transpose operation and $\mathbf{w}_{r}=\frac{\mathbf{g}_{0}}{\left\|\mathbf{g}_{0}\right\|}$ as stated in $[19,20]$.

From (2) and (3), the received signal-to-noise ratio (SNR) of the primary signal at the PR and the SU can be respectively derived as

$$
\mathrm{SNR}_{\mathrm{PR}}=\frac{P_{p}}{\sigma^{2}}\left|h_{0}\right|^{2} \text { and } \mathrm{SNR}_{\mathrm{SU}}=\frac{P_{p}}{\sigma^{2}}\left\|\mathbf{g}_{0}\right\|^{2} .
$$

Correspondingly, the outage probability for a given target rate $R_{p}$ between the PU and the PR is represented as

$$
\operatorname{Pr}\left\{O_{P, R}\right\}=\operatorname{Pr}\left\{\log _{2}\left(1+\mathrm{SNR}_{\mathrm{PR}}\right)<R_{p}\right\}=1-\exp \left(-\frac{\Delta_{0}}{\bar{\gamma}_{0}}\right),
$$

where $\bar{\gamma}_{0}=\frac{P_{p}}{d_{\mathrm{PU}, \mathrm{PR}}^{\alpha} \sigma^{2}}$ and $\Delta_{0}=2^{R_{p}}-1$.
Similarly, the outage probability between the PU and the $\mathrm{SU}$ is given by

$\operatorname{Pr}\left\{O_{P, S}\right\}=\operatorname{Pr}\left\{\log _{2}\left(1+\mathrm{SNR}_{\mathrm{SU}}\right)<R_{p}\right\}=\frac{\gamma\left(M, \Delta_{0} / \bar{\gamma}_{1}\right)}{\Gamma(M)}$,

where $\bar{\gamma}_{1}=\frac{P_{p}}{d_{\mathrm{PU}, \mathrm{SU}}^{\alpha} \sigma^{2}}, \gamma(\cdot, \cdot)$ and $\Gamma(\cdot)$ denote the lower incomplete gamma function and gamma function [21], respectively.

\subsection{Queueing stability}

In a communication network, the stability of the queue is a fundamental performance metric. Stability is defined as the state where all the queues in the network are stable. According to Rao and Ephremides [22], a queue is stable if and only if there exists a positive probability for the queue being empty, i.e.,

$$
\lim _{t \rightarrow \infty} \operatorname{Pr}\left\{Q_{i}(t)=0\right\}>0,
$$

where $Q_{i}(t)$ denotes the size of the $i$ th queue at time $t$. For a more rigorous definition of stability, we can refer to $[22,23]$. If the arrival and departure rates of a queueing system are stationary, the stability can be checked by using Loynes' theorem [24]. The theorem states that the queue is strictly stationary which requires the average arrival rate less than the average departure rate of the queue, i.e., the service rate. Otherwise, the queue is unstable.

\section{Cognitive cooperative protocol design with multiple antennas}

In this section, we investigate a novel cognitive cooperative access protocol which can efficiently combine the principles of cognitive radio with multiple antenna technology. From higher network layer viewpoint, we focus on analyzing the maximum stable throughput for both the PU and SU by using the queuing theory. Furthermore, we assume that the SU has perfect spectral sensing ability and mainly analyze the impact of multiple antennas on the cooperative access protocol for the PU and the SU in the article. The impact of imperfect spectral sensing problems is beyond the scope of our consideration.

\subsection{Non-cooperative access scheme}

In order to evaluate the gains obtained from the cooperative access scheme, the non-cooperative access scheme is analyzed as a baseline scenario, in which the PU transmits its data through the primary link directly without getting any assistance from the SU. The queue size of the PU or SU at time $t$, denoted by $Q_{i}(t), i \in\{p, s\}$, evolves as follows:

$$
Q_{i}(t)=\left(Q_{i}(t-1)-Y_{i}(t)\right)^{+}+X_{i}(t),
$$

where $X_{i}(t)$ represents the number of packet arrivals at time $t$ and is a Bernoulli stationary process with finite 
mean $\mathrm{E}\left[X_{i}(t)\right]=\lambda_{i} . Y_{i}(t)$ denotes the number of packet departures from the queue $Q_{i}$ at time $t$. The values of $X_{i}(t)$ and $Y_{i}(t)$ are taken from $\{0,1\}$. The function $(\cdot)^{+}$is defined as $(x)^{+}=\max (x, 0)$.

In the non-cooperative access scheme, the service process of the PU can be modeled as $Y_{p}(t)=\mathbf{1}\left[\bar{O}_{P, R}^{t}\right]$, where $\mathbf{1}[\cdot]$ is the indicator function, $\bar{O}_{a, b}^{t}$ denotes the complement of the outage event for link $a \rightarrow b$ at time $t$. From the definition of the service process, the average service process of the $\mathrm{PU}$ is given by

$$
\mu_{\mathrm{PN}}^{\max }=\mathrm{E}\left[Y_{P}(t)\right]=\operatorname{Pr}\left(\bar{O}_{P, R}^{t}\right)=\exp \left(-\frac{\Delta_{0}}{\bar{\gamma}_{0}}\right) .
$$

Due to assuming perfect spectral sensing at the SU, the PU's queue and the SU's queue are not interacting. Therefore, according to Loynes' theorem, the average service process is also defined as the maximum stable throughput of the corresponding queue. Hence, the stability of the PU's queue, under the non-cooperative access scheme, should satisfy

$$
\lambda_{p}<\mu_{\mathrm{PN}}^{\max }=\exp \left(-\frac{\Delta_{0}}{\bar{\gamma}_{0}}\right) .
$$

According to the principles of cognitive radio, the SU is allowed to access the licensed spectrum without producing the interference to the PR. Therefore, the SU can access the licensed spectrum when the PU does not have any packet in its queue. We can model the service process of the SU as $[13,14]$

$$
Y_{S}(t)=\mathbf{1}\left[\left\{Q_{p}(t)=0\right\} \cap \bar{O}_{S, R}^{t}\right],
$$

where $\left\{Q_{p}(t)=0\right\}$ represents the event that the PU's queue is empty at time $t$. According to Little's theorem $[24]$, it has probability $\operatorname{Pr}\left[Q_{p}(t)=0\right]=1-\lambda_{p} / \mu_{\mathrm{PN}}^{\max }$. Hence, the average service process of the $\mathrm{SU}$ is given by

$\mu_{\mathrm{SN}}^{\max }=\mathrm{E}\left[Y_{s}(t)\right]=\left(1-\frac{\lambda_{p}}{\mu_{\mathrm{PN}}^{\max }}\right)\left(1-\frac{\gamma\left(M, \Delta_{1} / \bar{\gamma}_{2}\right)}{\Gamma(M)}\right)$,

where $\Delta_{1}=2^{R_{s}}-1, \bar{\gamma}_{2}=\frac{P_{s}}{d_{\mathrm{SU}, \mathrm{SR}}^{\alpha} \sigma^{2}}$. Correspondingly, the stability of the SU's queue, under the non-cooperative access scheme, requires

$$
\lambda_{s}<\mu_{\mathrm{SN}}^{\max }=\left(1-\frac{\lambda_{p}}{\mu_{\mathrm{PN}}^{\max }}\right)\left(1-\frac{\gamma\left(M, \Delta_{1} / \bar{\gamma}_{2}\right)}{\Gamma(M)}\right) .
$$

\subsection{Cooperative access scheme}

In this section, we investigate a cognitive cooperative access scheme, in which the $\mathrm{SU}$ acts as a relay to deliver the PU's packets unsuccessfully received by the PR through the primary link directly. Therefore, in contrast to the non-cooperative access scheme, the PU's packet is removed from its queue whenever it is correctly received by the PR or the SU. Moreover, the conventional noncooperative ACK/NACK mechanism should be revised and the SU is allowed to send an ACK to notify the PU for successful decoding the packet [11-13]. To get more access opportunities, the SU simultaneously relays the PU's packets and transmits its own packets based on the superposition coding in the cooperative access scheme. Further, to concurrent relay the PU's packets and transmit its own packets based on the superposition coding such that they are received without interference at the PR and SR respectively, we design two $M \times 1$ weight vectors at the SU to the PU's signal and the SU's signal, i.e., $\mathbf{w}_{p}$ and $\mathbf{w}_{S}$, respectively. Thus, the received signal at the PR and SR can be represented as

$$
\begin{aligned}
& y_{\mathrm{PR}}^{S}=\sqrt{P_{s}} \mathbf{f}_{1}^{\dagger}\left(\sqrt{\beta} \mathbf{w}_{p} x_{p}+\sqrt{1-\beta} \mathbf{w}_{s} x_{s}\right)+n_{\mathrm{PR}}, \\
& y_{\mathrm{SR}}^{S}=\sqrt{P_{s}} \mathbf{f}_{0}^{\dagger}\left(\sqrt{1-\beta} \mathbf{w}_{s} x_{s}+\sqrt{\beta} \mathbf{w}_{p} x_{p}\right)+n_{\mathrm{SR}},
\end{aligned}
$$

where $\beta(0 \leq \beta \leq 1)$ denotes the power allocation factor, i.e., the parameter of the superposition coding. In order to eliminate the interference signal and maximize the desired signal at the PR and SR, we adopt the zero-forcing algorithm to design the weight vectors for simplicity. Then, two weight vectors $\mathbf{w}_{p}$ and $\mathbf{w}_{s}$ can be obtained by the following optimization problems $[15,25]$

$$
\begin{aligned}
\tilde{\mathbf{w}}_{p} & =\arg \max _{\mathbf{w}_{p}}\left|\mathbf{f}_{1}^{\dagger} \mathbf{w}_{p}\right| \\
\text { s.t. }\left|\mathbf{f}_{0}^{\dagger} \mathbf{w}_{p}\right| & =0 \text { and }\left\|\mathbf{w}_{p}\right\|=1 . \\
\tilde{\mathbf{w}}_{s} & =\arg \max _{\mathbf{w}_{s}}\left|\mathbf{f}_{0}^{\dagger} \mathbf{w}_{s}\right| \\
\text { s.t. }\left|\mathbf{f}_{1}^{\dagger} \mathbf{w}_{s}\right| & =0 \text { and }\left\|\mathbf{w}_{s}\right\|=1 .
\end{aligned}
$$

It seems difficult to solve the two optimization problems to get the zero-forcing beamforming weight vectors $\mathbf{w}_{p}$ and $\mathbf{w}_{s}$. In fact, we can use projection matrix theory [26] to get the optimum weight vectors as follows:

$$
\widetilde{\mathbf{w}}_{p}=\frac{\mathbf{V}_{\mathrm{PR}}^{\perp} \mathbf{f}_{1}}{\left\|\mathbf{V}_{\mathrm{PR}}^{\perp} \mathbf{f}_{1}\right\|} \text { and } \widetilde{\mathbf{w}}_{s}=\frac{\mathbf{V}_{\mathrm{SR}}^{\perp} \mathbf{f}_{0}}{\left\|\mathbf{V}_{\mathrm{SR}}^{\perp} \mathbf{f}_{0}\right\|},
$$

where $\mathbf{V}_{\mathrm{PR}}^{\perp}$ and $\mathbf{V}_{\mathrm{SR}}^{\perp}$ are the projection matrices for the PR and $\mathrm{SR}$ and given, respectively, by

$$
\mathbf{V}_{\mathrm{PR}}^{\perp}=\left(\mathbf{I}-\mathbf{f}_{0}\left(\mathbf{f}_{0}^{\dagger} \mathbf{f}_{0}\right)^{-1} \mathbf{f}_{0}^{\dagger}\right) \text { and } \mathbf{V}_{\mathrm{SR}}^{\perp}=\left(\mathbf{I}-\mathbf{f}_{1}\left(\mathbf{f}_{1}^{\dagger} \mathbf{f}_{1}\right)^{-1} \mathbf{f}_{1}^{\dagger}\right) \text {. }
$$


To this end, the received SNR at the PR and the SR can be calculated from (14)-(17), after some algebraic manipulations, as

$$
\mathrm{SNR}_{\mathrm{PR}}^{S}=\frac{P_{s} \beta}{\sigma^{2}}\left\|\mathbf{V}_{\mathrm{PR}}^{\perp} \mathbf{f}_{1}\right\|^{2} \text { and } \mathrm{SNR}_{\mathrm{SR}}^{S}=\frac{P_{s}(1-\beta)}{\sigma^{2}}\left\|\mathbf{V}_{\mathrm{SR}}^{\perp} \mathbf{f}_{0}\right\|^{2} .
$$

To derive the maximum stable throughput for the primary system and the secondary system under the cognitive cooperative access scheme, we first present the following theorem $[15,25]$.

Theorem 1. Let $\mathbf{h}_{0} \sim \mathcal{C N}_{M, 1}\left(\mathbf{0}_{M}, \mathbf{I}_{M}\right), \mathbf{h}_{1} \sim \mathcal{C N}_{M, 1}$ $\left(\mathbf{0}_{M}, \mathbf{I}_{M}\right)$ and $\mathbf{V}^{\perp}=\left(\mathbf{I}-\mathbf{h}_{1}\left(\mathbf{h}_{1}^{\dagger} \mathbf{h}_{1}\right)^{-1} \mathbf{h}_{1}^{\dagger}\right)$. Then $\left\|\mathbf{V}^{\perp} \mathbf{h}_{0}\right\|^{2}$ is Chi-square distributed with $2(M-1)$ degrees of freedom, and its probability density function is given as

$$
f(x)=\frac{x^{M-2}}{(M-2) !} e^{-x}, \quad x \geq 0 .
$$

In what follows, we analyze the maximum stable throughput of the cognitive cooperative access scheme and compare with that of the non-cooperative access scheme.

\subsubsection{PU stability}

In contrast to the non-cooperative access scheme, the SU has two queues in the cognitive cooperative access scheme, i.e., one queue $Q_{s}$ storing its own packets and one queue $Q_{s p}$ containing the packets from the PU which are not successfully received by the PR. Hence, the packets in the queue $Q_{p}$ will be removed in the cooperative access scheme whenever the packets are successfully received by the PR or the SU, which means that the maximum stable throughput of the PU depends on both the stability of the queue $Q_{p}$ and $Q_{s p}$. Hence, the service process of the PU can be modeled as

$$
Y_{p}(t)=\mathbf{1}\left[\bar{O}_{P, R}^{t}\right]+\mathbf{1}\left[O_{P, R}^{t} \cap \bar{O}_{P, S}^{t}\right] .
$$

Then, the average of the service process of the PU in the cognitive cooperative access scheme can be derived as

$$
\begin{aligned}
\mu_{P C}^{\max }= & \mathrm{E}\left[Y_{p}(t)\right] \\
= & \operatorname{Pr}\left(\bar{O}_{P, R}^{t}\right)+\left(1-\operatorname{Pr}\left(O_{P, R}^{t}\right)\right) \operatorname{Pr}\left(\bar{O}_{P, S}^{t}\right) \\
= & \exp \left(-\frac{\Delta_{0}}{\bar{\gamma}_{0}}\right)+\left(1-\exp \left(-\frac{\Delta_{0}}{\bar{\gamma}_{0}}\right)\right) \\
& \times\left(1-\frac{\gamma\left(M, \Delta_{0} / \bar{\gamma}_{1}\right)}{\Gamma(M)}\right) .
\end{aligned}
$$

In what follows, we analyze the stability of the queue $Q_{s p}$. The evolution of the queue $Q_{s p}$ can also be modeled as

$$
Q_{s p}(t)=\left(Q_{s p}(t-1)-Y_{s p}(t)\right)^{+}+X_{s p}(t),
$$

where $Y_{s p}(t)=\mathbf{1}\left[\left\{Q_{p}(t)=0\right\} \cap \bar{O}_{S, P}^{t}(\beta)\right]$ denotes the number of packet departures from the queue $Q_{s p}$ and $X_{s p}(t)=\mathbf{1}\left[\left\{Q_{p}(t) \neq 0\right\} \cap \bar{O}_{P, S}^{t} \cap O_{P, R}^{t}\right]$ is the number of packet arrivals of the queue $Q_{s p}$ at time $t$, respectively. It is worth noting from (23) that only the queue $Q_{p}$ is empty, the $\mathrm{SU}$ will access the channel to transmit its own packets and the packets in the queue $Q_{s p}$ based on the superposition coding to get more access opportunities.

Correspondingly, the average packet arrival rate and departure rate for the queue $Q_{s p}$, at time $t$, can be respectively computed as

$$
\begin{aligned}
\lambda_{s p}= & \mathrm{E}\left[X_{s p}(t)\right]=\frac{\lambda_{p}}{\mu_{P C}^{\max }}\left(1-\frac{\gamma\left(M, \Delta_{0} / \bar{\gamma}_{1}\right)}{\Gamma(M)}\right) \\
& \times\left(1-\exp \left(-\frac{\Delta_{0}}{\bar{\gamma}_{0}}\right)\right) . \\
\mu_{S P}^{\max }= & \mathrm{E}\left[Y_{s p}(t)\right]=\left(1-\frac{\lambda_{p}}{\mu_{P C}^{\max }}\right)\left(1-\frac{\gamma\left(M-1, \Delta_{0} / \bar{\gamma}_{3}\right)}{\Gamma(M-1)}\right),
\end{aligned}
$$

where $\bar{\gamma}_{3}=\frac{P_{s} \beta}{d_{\mathrm{SU}, \mathrm{PR}}^{\alpha} \sigma^{2}}$. To this end, according to Loynes' theorem [23], the stability condition of the PU's queue in the proposed cooperative access scheme can be derived from (22)-(25) as

$$
\begin{aligned}
\lambda_{p} & <\mu_{C}^{\max } \\
& =\frac{u_{P C}^{\max } \times\left(1-\frac{\gamma\left(M-1, \Delta_{0} / \bar{\gamma}_{3}\right)}{\Gamma(M-1)}\right)}{\left(1-\exp \left(-\frac{\Delta_{0}}{\bar{\gamma}_{0}}\right)\right)\left(1-\frac{\gamma\left(M, \Delta_{0} / \bar{\gamma}_{1}\right)}{\Gamma(M)}\right)+\left(1-\frac{\gamma\left(M-1, \Delta_{0} / \bar{\gamma}_{3}\right)}{\Gamma(M-1)}\right)} .
\end{aligned}
$$

\subsubsection{SU stability}

According to the cognitive cooperative access scheme, the service process of the SU can be modeled as

$$
\begin{aligned}
Y_{s}(t)= & \mathbf{1}\left[\left\{Q_{p}(t)=0\right\} \cap\left\{Q_{s p}(t)=0\right\} \cap \bar{O}_{S, R}^{t}\right] \\
& +\mathbf{1}\left[\left\{Q_{p}(t)=0\right\} \cap\left\{Q_{s p}(t) \neq 0\right\} \cap \bar{O}_{S, R}^{t}(\beta)\right],
\end{aligned}
$$

where $\left\{Q_{s p}(t)=0\right\}$ denotes the event that the queue $Q_{s p}$ is empty at time $t$ and the probability of the empty of the queue $Q_{s p}$ based on Little's theorem [24] is given by

$$
\operatorname{Pr}\left[Q_{s p}(t)=0\right]=1-\frac{\lambda_{s p}}{\mu_{S P}^{\max }} .
$$


For the empty of the queue $Q_{s p}$ condition, the SU only establishes a communication between itself and the SR. From (27), the average service rate of the SU can be derived as

$$
\begin{aligned}
\mu_{S C}^{\max }= & \mathrm{E}\left[Y_{s}(t)\right] \\
= & \left(1-\frac{\lambda_{p}}{\mu_{P C}^{\max }}\right)\left(1-\frac{\lambda_{s p}}{\mu_{S P}^{\max }}\right)\left(1-\frac{\gamma\left(M, \Delta_{1} / \bar{\gamma}_{2}\right)}{\Gamma(M)}\right) \\
& +\left(1-\frac{\lambda_{p}}{\mu_{P C}^{\max }}\right) \frac{\lambda_{s p}}{\mu_{S P}^{\max }}\left(1-\frac{\gamma\left(M-1, \Delta_{1} / \bar{\gamma}_{4}\right)}{\Gamma(M-1)}\right),
\end{aligned}
$$

where $\bar{\gamma}_{4}=\frac{P_{S}(1-\beta)}{d_{\mathrm{S} U \mathrm{SR}}^{\alpha} \sigma^{2}}$.

Using Loynes' theorem [23], the stability condition of the SU's queue in the cognitive cooperative access scheme requires

$$
\begin{aligned}
\lambda_{s}< & \left(1-\frac{\lambda_{p}}{\mu_{P C}^{\max }}\right)\left(1-\frac{\lambda_{s p}}{\mu_{S P}^{\max }}\right)\left(1-\frac{\gamma\left(M, \Delta_{1} / \bar{\gamma}_{2}\right)}{\Gamma(M)}\right) \\
& +\left(1-\frac{\lambda_{p}}{\mu_{P C}^{\max }}\right) \frac{\lambda_{s p}}{\mu_{S P}^{\max }}\left(1-\frac{\gamma\left(M-1, \Delta_{1} / \bar{\gamma}_{4}\right)}{\Gamma(M-1)}\right) .
\end{aligned}
$$

\section{Cooperative gains and parameters optimization}

\subsection{Cooperative gains}

In this section, we first analyze the cooperative gains for the PU and the SU in the cognitive cooperative access scheme, respectively. In what follows, we define the maximum stable throughput cooperative gains for the PU and the $\mathrm{SU}$ as

$$
G_{p}(M, \beta)=\mu_{C}^{\max }-\mu_{P N}^{\max } \text { and } G_{S}(M, \beta)=\mu_{S C}^{\max }-\mu_{S N}^{\max } .
$$

Theorem 2. The upper bounds of the maximum stable throughput cooperative gains for both the PU and the SU are, respectively, given by

$$
G_{p}^{\text {bound }}=\frac{1}{2-\mu_{P N}^{\max }}-\mu_{P N}^{\max } \text { and } G_{s}^{\text {bound }}=\lambda_{p}\left(\frac{1}{\mu_{P N}^{\max }}-1\right) .
$$

Proof. In case where $M$ grows asymptotically large, we have

$$
\lim _{M \rightarrow \infty} \frac{\gamma(M, x)}{\Gamma(M)}=0 .
$$

From (26) and (33), the maximum stable throughput of the PU with cooperation can be derived as

$$
\lim _{M \rightarrow \infty} \mu_{C}^{\max }=\frac{1}{1+\left(1-\mu_{P N}^{\max }\right)} .
$$

Since the maximum stable throughput of the PU without cooperation is independent of $M$, it keeps constant when $M$ grows asymptotically large. To this end, the cooperative gain bound of the PU can be derived as (32).

By following the similar proof for that of the PU, the cooperative gain bound of the SU can be obtained after some simple mathematical manipulations.

\subsection{Parameters optimization}

Due to the fact that the parameter of superposition coding, i.e., the power allocation factor $\beta$, introduces an interesting tradeoff between the maximum stable throughput of the PU and the SU. Hence, we optimize the appropriate power allocation factor $\beta$ to maximize the stable throughput of the SU while supporting the pre-selected PU's stable throughput $\lambda_{p 0}\left(\lambda_{p 0}<\mu_{P N}^{\max }\right)$ in this section. The optimization problem can be formulated as

$$
\begin{aligned}
&(\beta, M)_{\mathrm{opt}}=\arg \max _{\beta}\left\{\lambda_{s}(\beta, M)\right\} \\
& \text { s.t. } \quad \lambda_{p}(M, \beta) \geq \lambda_{p 0}, \\
& 0 \leq \beta \leq 1, \\
&\left\{M \in Z^{+} \& M \geq 2\right\} .
\end{aligned}
$$

It is worth pointing out that the solution of the optimization problem should satisfy the inequality $\lambda_{p}(M, \beta) \geq$ $\lambda_{p 0}$. Since it is difficult to obtain a closed form expression for $\beta_{\text {opt }}$, we numerically evaluate $\beta_{\text {opt }}$ by Monte Carlo simulations. As shown in Figure 2, the stable throughput of the SU decreases with the power allocation factor $\beta$ whereas the stable throughput of the PU presents the flat behavior when the power allocation factor $\beta$ exceeds a certain value. Hence, the optimal power allocation factor $\beta_{\text {opt }}$ can always be found for all practical SNR values under different antenna configurations through simulations. Besides, note that the use of $\beta_{\text {opt }}$ and $M_{\text {opt }}$ based on (35), will simultaneously (i) achieve a significant performance improvement for the stable throughput at both the PU and the SU, and (ii) guarantee that the PU's stable throughput of the cooperative access scheme will equal to or outperform the PU's stable throughput of the non-cooperative scheme.

4.3 Numerical results for stable throughput performance In this section, we make extensive numerical results to evaluate the performance of the proposed cooperative access scheme. To present the advantage of the proposed cooperative access scheme, the existing cooperative access scheme based on DPC in [13] is provided for comparison in the simulations. Throughout the article, a system topology where the PU, SU, and PR are collinear is considered. Unless otherwise specify, the simulation parameters 


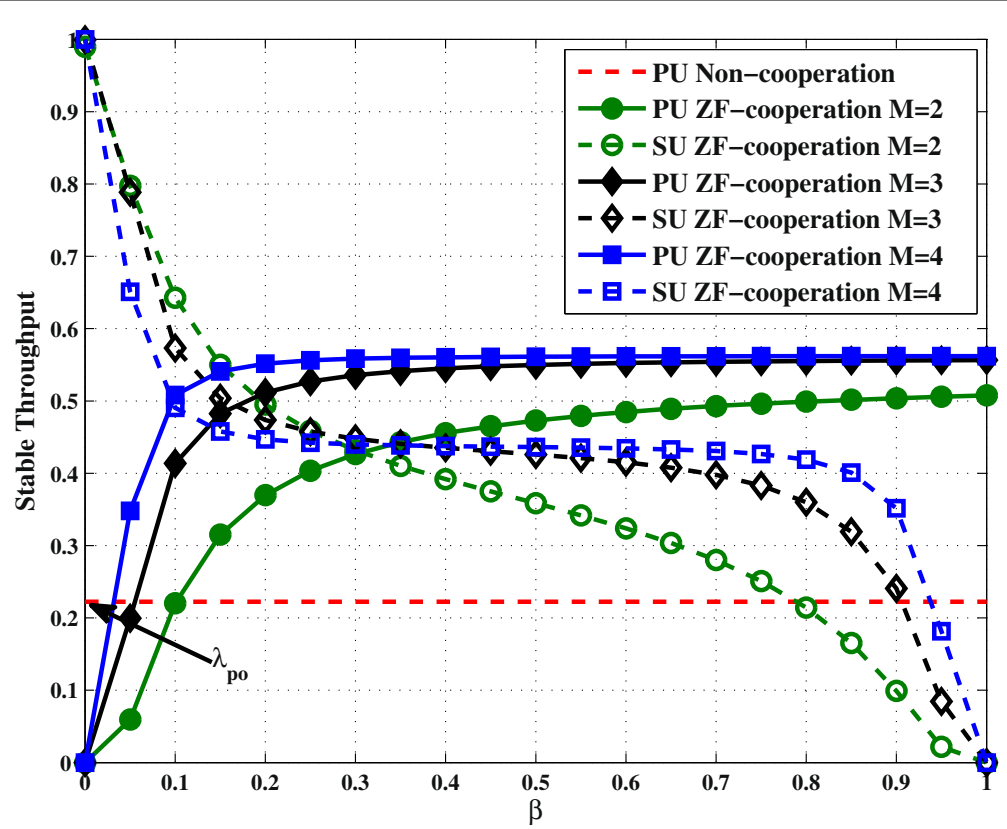

Figure 2 Maximum stable throughput for $\mathrm{PU}$ and $\mathrm{SU}$ versus $\beta$ with different antenna configurations when $P_{p}=3 \mathrm{~dB}, P_{s}=5 \mathrm{~dB}$, and $d=0.4$.

are set as follows: $d_{\mathrm{PU}, \mathrm{PR}}=1, d_{\mathrm{PU}, \mathrm{SU}}=1-d, d_{\mathrm{SU}, \mathrm{PR}}=d$, $d_{\mathrm{SU}, \mathrm{SR}}=d, \sigma^{2}=1, \alpha=2, R_{p}=2$, and $R_{s}=2$.

Figure 3 compares the maximum stable throughput of cooperative and non-cooperative access schemes as a function of the PU's arrival rate $\lambda_{p}$ using the fixed power allocation factor $\beta=0.5$. We label the proposed cooperative access scheme based on zero-forcing algorithm as ZF-cooperation. It is noted from the figure that the proposed cooperative access scheme outperforms its noncooperative counterpart. Further, as can be expected in any conventional multiple antennas system since the extra number of antennas provides additional space diversity gain, the maximum stable throughput of the $\mathrm{SU}$ is significantly improved in the proposed cooperative access

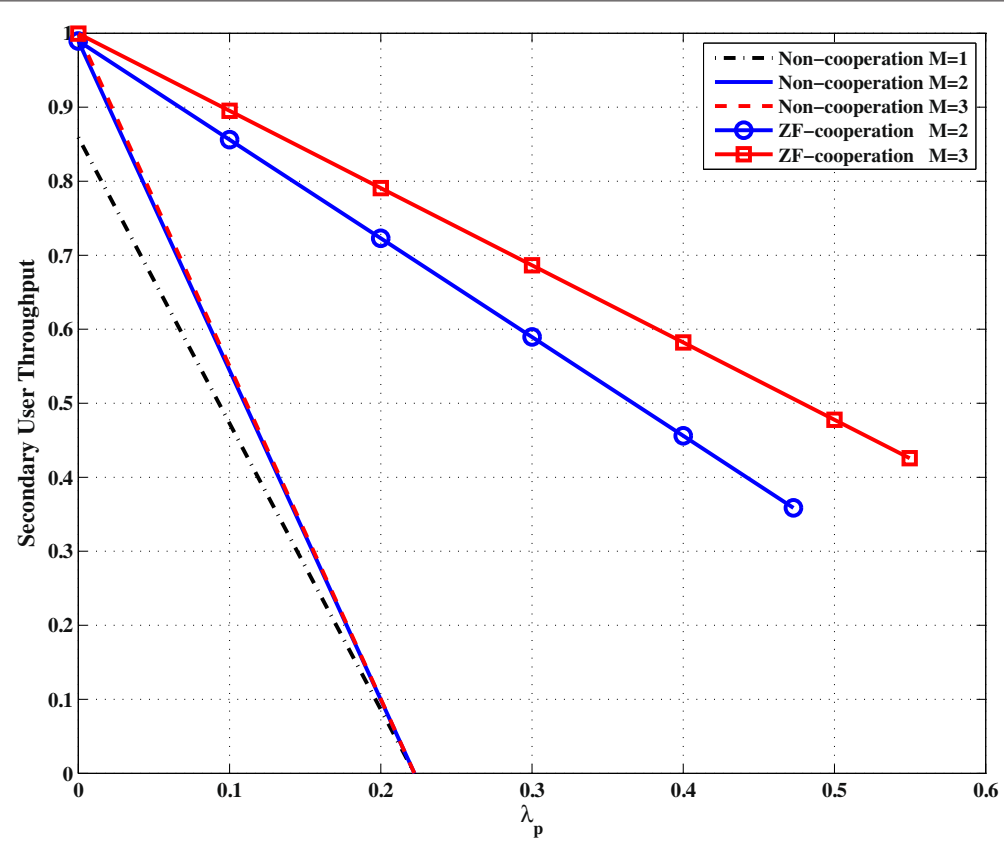

Figure 3 Maximum stable throughput for SU versus $\lambda_{p}$ with different antenna configurations when $P_{p}=3 \mathrm{~dB}, P_{s}=5 \mathrm{~dB}$, and $d=0.4$. 
scheme. However, in contrast to the cooperative access scheme, increasing the number of antennas at the SU does not provide any diversity gain in the non-cooperative access scheme due to the restriction of access opportunities for the SU. Hence, increasing the number of antennas only provides the additional coding gain for the $\mathrm{SU}$ in the non-cooperative access scheme.

In Figure 4, we compare the proposed cooperative access scheme, i.e., ZF-cooperation, with the conventional cooperative access scheme by using DPC in [13] (labeled as DPC-cooperation). We adopt the selection algorithm for the parameter of superposition coding in [13], in which the power allocation factor $\beta$ is selected from the PU's point of view. Hence, we select $\beta_{0}=0.6$ and $\beta_{1}=0.7$ for the ZF-cooperation scheme and the DPC-cooperation scheme, respectively. To a fair comparison between the two schemes, we assume that for ZF-cooperation scheme the PU and SU transmit with power $P_{p}$ and $P_{s}$, respectively, while for the DPC-cooperation scheme the PT and SU transmit with power $P_{p}$ and $\frac{P_{s}}{2}$, respectively. This is due to the fact that two SUs are selected to transmit its own packets and relay the PU's packets in the DPC-cooperation scheme. As can be observed in the figure, the proposed ZF-cooperation scheme significantly outperforms the DPC-cooperation scheme for both primary and secondary stable throughput. Furthermore, the non-cooperative access scheme achieves better performance than that of the DPC-cooperation scheme under low SNR regime. This is can be explained by the fact that the DPC-cooperation scheme introduces a severe interference to the primary transmission in the low SNR regime, which results in a lower gain throughput for the SU. However, different from the DPC-cooperation scheme, there exists no interference at the $\mathrm{PU}$ and $\mathrm{SU}$ in all SNR region in the proposed ZF-cooperation scheme.

Figure 5 illustrates the impact of the number of antennas $M$ on the maximum PU's stable throughput performance when $P_{s}=5$ and $P_{p}=1,3,5 \mathrm{~dB}$, respectively. As can be expected, when the transmit power of the PU increases, the maximum PU's stable throughput is significantly improved. It is also observed that the maximum PU's stable throughput of the proposed ZF-cooperation scheme is improved as the number of antennas $M$ increases due to the fact that a higher diversity gain can be obtained by increasing the number of antenna. However, the maximum PU's stable throughput almost keeps constant when $M \geq 6$ under different transmit powers of the PU, which implies that an adequate number of antennas can be selected for the design of the system to reduce the complexity of our scheme.

Figure 6 plots the cooperative gain of the PU versus the transmit power $P_{s}$ when $P_{p}=1 \mathrm{~dB}$ and $3 \mathrm{~dB}$, respectively. It is noted from this figure that more cooperative gain of the PU can be obtained when the quality of PU's link is poor. In addition, we can observe that the cooperative gain can be gradually improved by increasing the transmit power $P_{S}$ of the SU and the number of antennas $M$. Specifically, when the number of antennas increases to a certain value, the full cooperative gain can be achieved

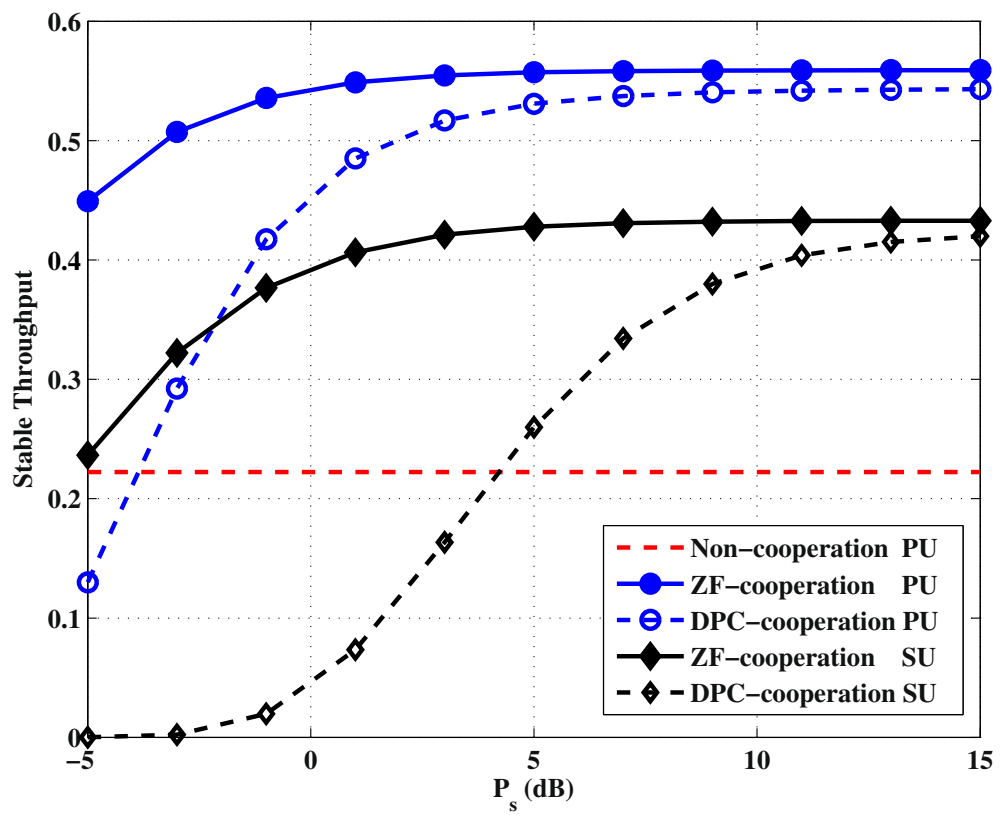

Figure 4 Maximum stable throughput for $\mathrm{PU}$ and SU versus $P_{s}$ when $M=3, P_{p}=3 \mathrm{~dB}$, and $d=0.4$. 


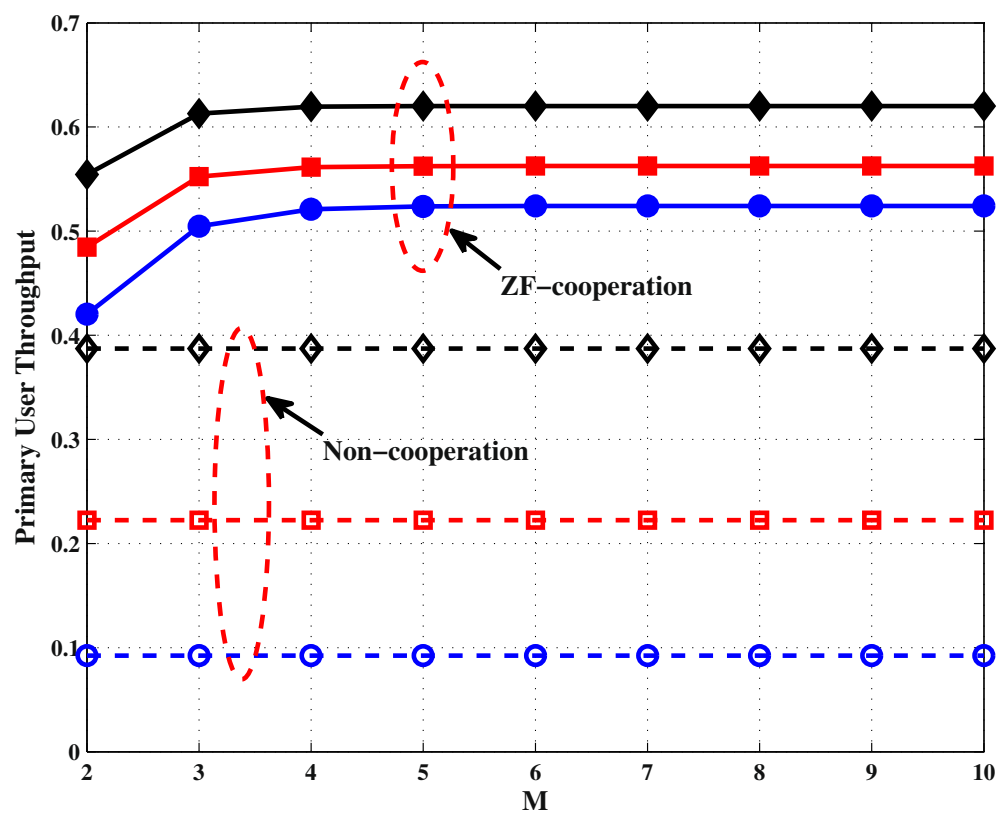

Figure 5 Maximum stable throughput for PU versus $M$ when $P_{s}=5 \mathrm{~dB}, P_{p}=1,3,5 \mathrm{~dB}$, and $d=0.4$.

in the proposed cooperative access scheme, which verifies our analysis in Section 4.

Figure 7 shows the impact of imperfect CSI on the PU and the SU performances of the proposed ZF-cooperation scheme. In previous analysis, we assume that perfect CSI about the links can be obtained at the SU. Such an assumption, however, does not hold in practical systems. The reason is that channel knowledge is always outdated due to the effect of delay and time-varying of the wireless link, which implies that we need to consider the impact of imperfect CSI. Since we are more interested in studying the impact of imperfect CSI on the designed weight vectors at the SU, we only consider the imperfect CSI about the channel $\mathbf{f}_{0}$ between the $S U$ and the $S R$ and the channel $\mathbf{f}_{1}$ between the $\mathrm{SU}$ and the PR in the simulation. The imperfect CSI
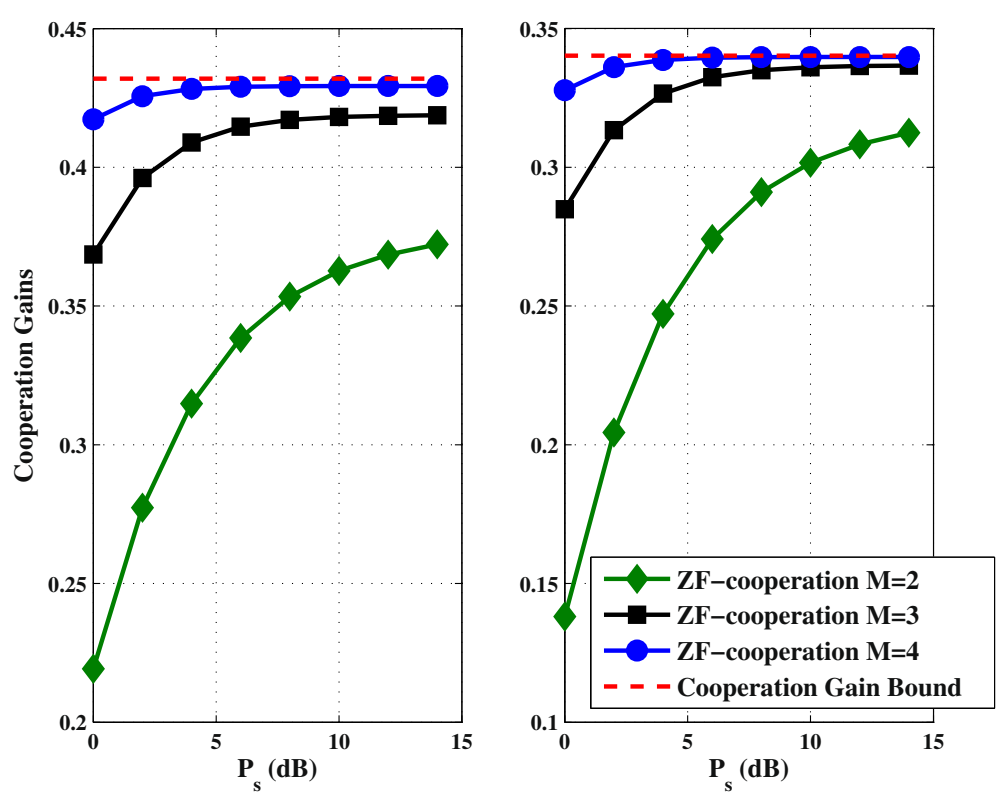

Figure 6 Cooperation gain for $\mathrm{PU}$ versus $P_{s}$ with different antenna configurations when $P_{p}=1,3 \mathrm{~dB}$ and $d=0.4$. 


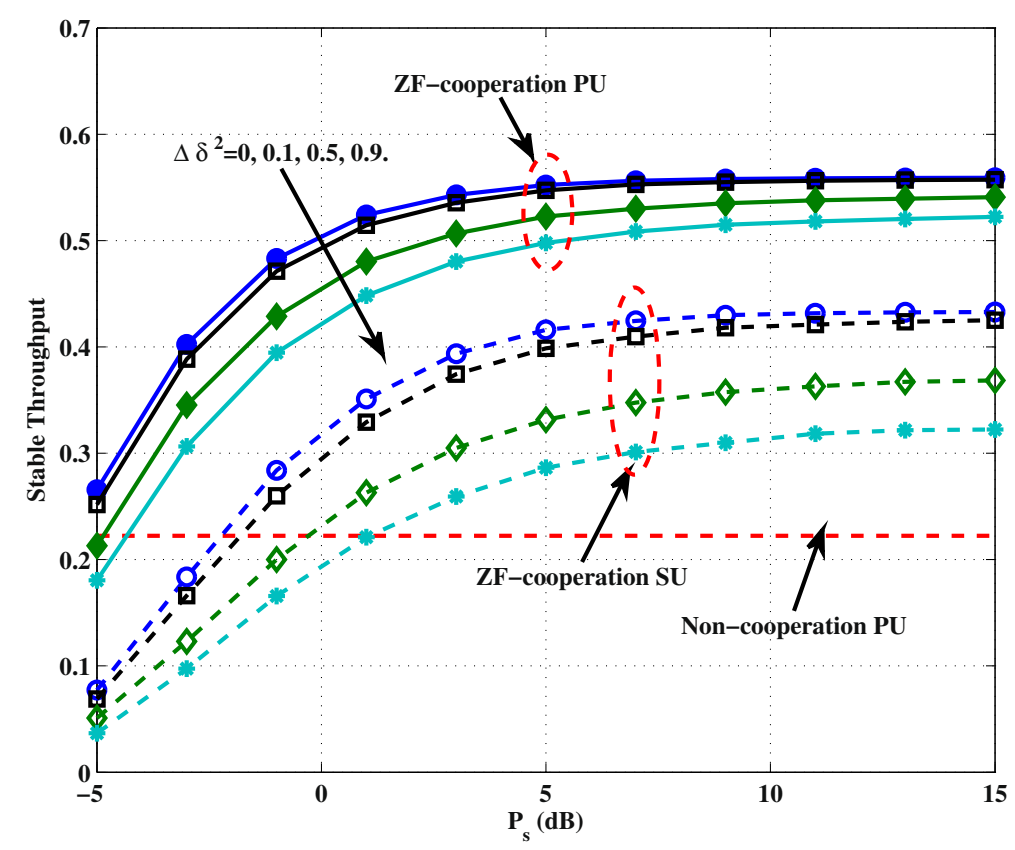

Figure 7 Maximum stable throughput for PU and SU versus $P_{s}$ under imperfect $\mathrm{CSI}$ when $M=3, P_{p}=3 \mathrm{~dB}$, and $d=0.4$.

can be described, using the correlation model [27-29], as follows:

$$
\begin{aligned}
& \mathbf{f}_{0}=\sqrt{1-\Delta \delta^{2} \widetilde{\mathbf{f}}_{0}}+\sqrt{\Delta \delta^{2}} \mathbf{n}_{0}, \\
& \mathbf{f}_{1}=\sqrt{1-\Delta \delta^{2} \tilde{\mathbf{f}}_{1}}+\sqrt{\Delta \delta^{2}} \mathbf{n}_{1},
\end{aligned}
$$

where $\widetilde{\mathbf{f}}_{0}$ denotes the outdated CSI between the SU and the SR, and $\widetilde{\mathbf{f}}_{1}$ is the outdated CSI between the SU and the PR. $\mathbf{n}_{0}$ and $\mathbf{n}_{1}$ are independent CSCG random vectors with each element having zero mean and unit variance, and $\Delta \delta^{2}\left(0 \leq \Delta \delta^{2} \leq 1\right)$ represents the variance of the outdated CSI. As shown in Figure 7, the stable throughput for the PU and the SU decreases for higher $\Delta \delta^{2}$. The result can be expected since increases of $\Delta \delta^{2}$ represent that the channel information about $\widetilde{\mathbf{f}}_{0}$ and $\widetilde{\mathbf{f}}_{1}$ becomes more inaccuracy at the SU. On the other hand, while the stable throughput of the PU decreases when the variance of the outdated CSI $\Delta \delta^{2}$ becomes larger, the proposed ZF-cooperation scheme still outperforms the non-cooperative access scheme, which means that the proposed ZF-cooperation scheme keeps robust against the imperfect CSI.

Figure 8 shows how the distance $d_{P U, S U}$ affects the maximum stable throughput for the PU and SU when $M=3$ and $P_{p}=P_{s}=1 \mathrm{~dB}$. From the figure, we see that the proposed cooperative access scheme achieves a better stable throughput performance than the noncooperative access scheme for the PU over all values of $d_{P U, S U}$. In addition, as we can observe, there exists an optimal $d_{P U, S U}$ to the maximum stable throughput of the
PU and the SU, respectively. For the proposed cooperative access scheme, we note that the PU's stable throughput improves for increasing $d_{P U, S U}$ when $0.1 \leq d_{\mathrm{PU}, \mathrm{SU}}<$ 0.7 , but gets worse when $0.7 \leq d_{\mathrm{PU}, \mathrm{SU}} \leq 0.9$. This is due to the fact that the PU's performance for the proposed cooperative access scheme is dominated by the worst link as the conventional relay networks. For $0.1 \leq$ $d_{\mathrm{PU}, \mathrm{SU}}<0.7$, the SU-PR link is the worst link compared to the PU-SU link, it has a lower SNR due to the longer distance. While for $0.7 \leq d_{\mathrm{PU}, \mathrm{SU}} \leq 0.9$, the longer distance results in the poor link between the PU and the SU.

\section{Delay analysis}

In this section, we analyze the average end-to-end delay of the PU in the proposed cooperative access scheme. Before delving into the details, we first give the definition of the end-to-end delay for a packet, which is described as the time from the packet arrives at the queue $Q_{i}$ till it is correctly received by the respective receiver.

\subsection{Non-cooperative access scheme}

According to Little's theorem, the average end-to-end delay of a packet in the queue $Q_{p}$ can be modeled as

$$
\bar{T}_{P N}=\frac{1}{\lambda_{p}} \lim _{T \rightarrow \infty} \frac{\sum_{t=1}^{T} Q_{p}(t)}{T}=\frac{\mathrm{E}\left[Q_{p}\right]}{\lambda_{p}},
$$

where $\mathrm{E}\left[Q_{p}\right]$ denotes the average queue size. 


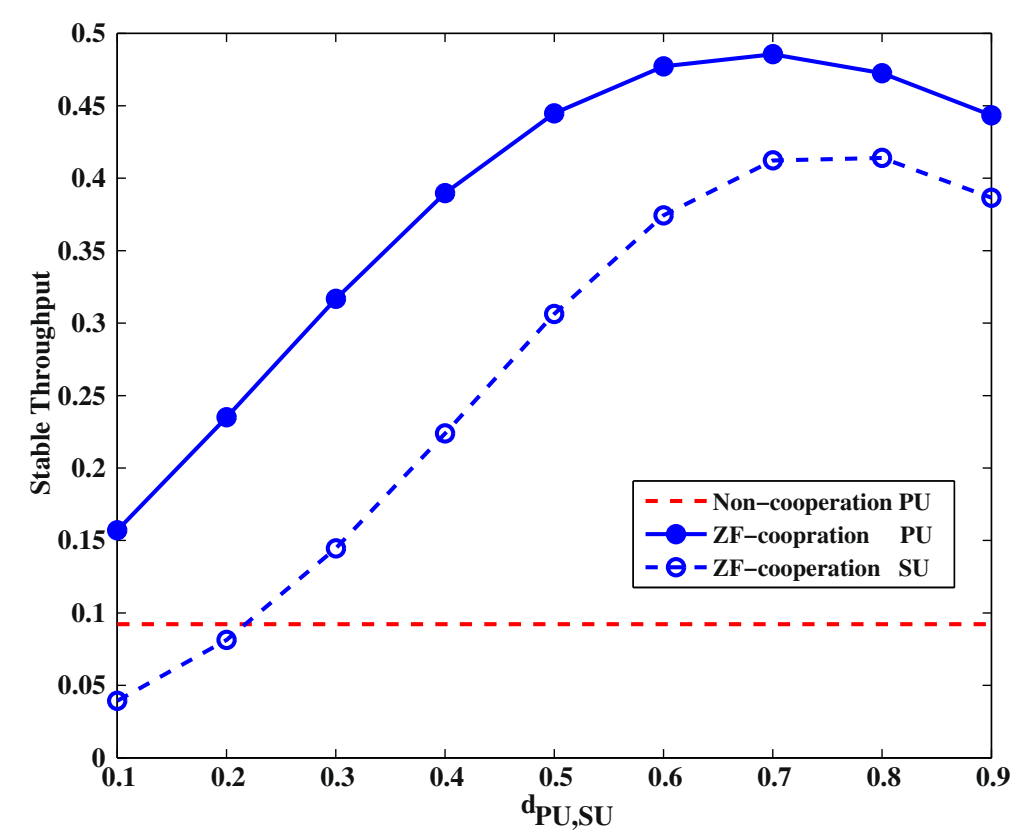

Figure 8 Maximum stable throughput for $\mathrm{PU}$ and SU versus $d_{P U}, S U$ when $M=3, P_{p}=P_{s}=1 \mathrm{~dB}$, and $d=0.4$.

Theorem 3. The average end-to-end delay for a packet of the PU in the non-cooperative access scheme is

$$
\bar{T}_{P N}=\frac{1-\lambda_{p}}{\mu_{P N}^{\max }-\lambda_{p}} .
$$

Proof. In order to analyze the average end-to-end delay of a packet in the corresponding queue $Q_{p}$, we first define two state transition probabilities as follows:

$$
\begin{aligned}
& \lambda_{p}^{n}=\operatorname{Pr}\left\{Q_{p}(t+1)=n+1 \mid Q_{p}(t)=n\right\}, \\
& \mu_{p}^{n}=\operatorname{Pr}\left\{Q_{p}(t+1)=n-1 \mid Q_{p}(t)=n\right\},
\end{aligned}
$$

where $Q_{q}(t)=n$ represents that the length of the queue, at time $t$, is $n$. As the queue evolution model in (8), we can get $\lambda_{p}^{n=0}=\lambda_{p}, \lambda_{p}^{n \geq 1}=\lambda_{p}\left(1-\mu_{P N}^{\max }\right)$ and $\mu_{p}^{n \geq 1}=\left(1-\lambda_{p}\right) \mu_{P N}^{\max }$. Therefore, the stable distribution of the queue $Q_{p}$ can be derived by solving the balance equation [23]. Define $q_{p}^{n}$ as $\operatorname{Pr}\left(Q_{p}=n\right)$, we have

$$
\begin{aligned}
& q_{p}^{0}=\left(1-\sum_{n=1}^{\infty} \prod_{k=0}^{n-1} \frac{\lambda_{p}^{k}}{\mu_{p}^{k+1}}\right)^{-1}=1-\frac{\lambda_{p}}{\mu_{P N}^{\max }}, \\
& q_{p}^{n}=q_{p}^{0} \prod_{k=0}^{n-1} \frac{\lambda_{p}^{k}}{\mu_{p}^{k+1}}=\frac{q_{p}^{0}}{\mu_{P N}^{\max }}\left(\frac{\lambda_{p}}{1-\lambda_{p}}\right)^{n}\left(\frac{1-\mu_{P N}^{\max }}{\mu_{P N}^{\max }}\right)^{n-1} .
\end{aligned}
$$

To this end, the average queue size of $Q_{p}$ can be derived as

$$
\mathrm{E}\left[Q_{p}\right]=\sum_{n=0}^{\infty} n \operatorname{Pr}\left(Q_{p}=n\right)=\frac{\lambda_{p}\left(1-\lambda_{p}\right)}{\mu_{P N}^{\max }-\lambda_{p}} .
$$

This completes the proof of Theorem 3.

\subsection{Cooperative access scheme}

For the proposed cooperative access scheme, the packet is dropped from the queue $Q_{p}$ as long as it is successfully received by the PR or the SU. Thus, the average end-toend delay of the PU's packet consists of two parts, one delay in the queue $Q_{p}$ and the other delay in the queue $Q_{s p}$.

Theorem 4. The average end-to-end delay for a packet of the PU in the proposed cooperative access scheme is

$$
\bar{T}_{P C}=\frac{1-\lambda_{p}}{\mu_{P C}^{\max }-\lambda_{p}}+\frac{1-\lambda_{s p}}{\mu_{S P}^{\max }-\lambda_{s p}}\left(1-\frac{\mu_{P N}^{\max }}{\mu_{P C}^{\max }}\right) .
$$

Proof. From the above analysis, we obtain that the total average end-to-end delay encountered by a packet in the PU's queue can be modeled as

$$
\bar{T}_{P C}= \begin{cases}\bar{T}_{P}, & \mathrm{~A} \\ \bar{T}_{P}+\bar{T}_{S P}, & \overline{\mathrm{A}}\end{cases}
$$

where $\bar{T}_{P}=\frac{1}{\lambda_{p}} \lim _{T \rightarrow \infty} \frac{\sum_{t=1}^{T} Q_{p}(t)}{T}=\frac{\mathrm{E}\left[Q_{p}\right]}{\lambda_{p}}$ and $\bar{T}_{S P}=\frac{1}{\lambda_{s p}}$ $\lim _{T \rightarrow \infty} \frac{\sum_{t=1}^{T} Q_{s p}(t)}{T}=\frac{\mathrm{E}\left[Q_{s p}\right]}{\lambda_{s p}}$. The event A denotes that a 


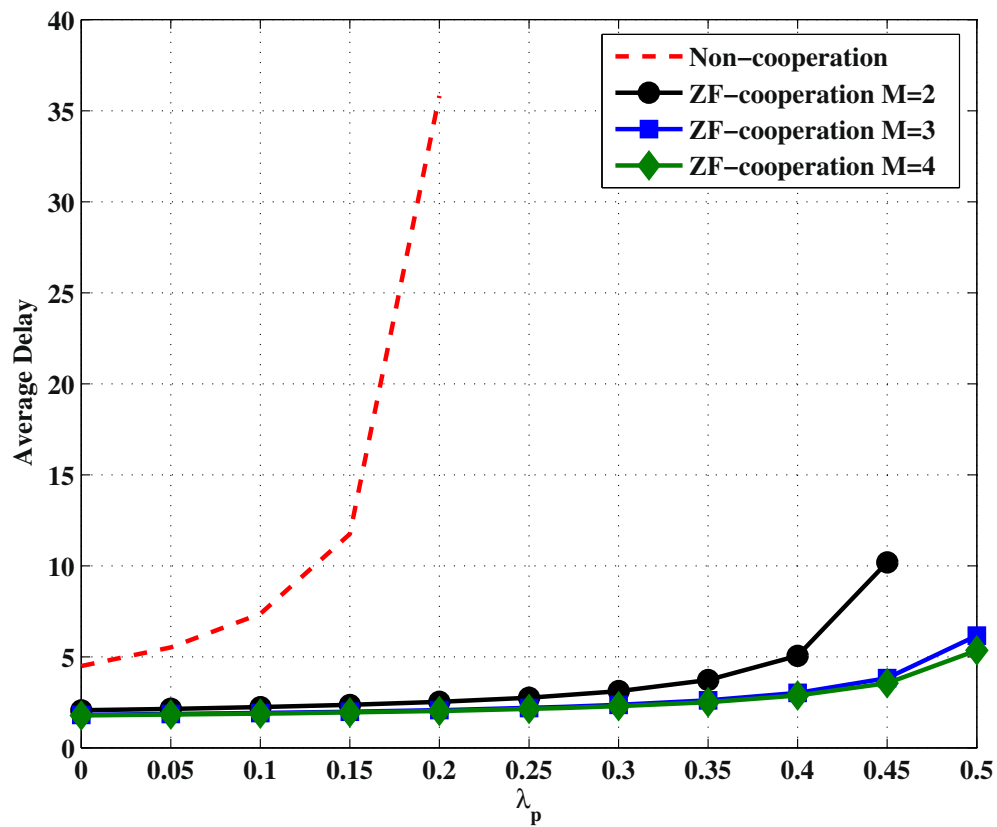

Figure 9 verage queue delay for PU versus $\lambda_{p}$ with different antenna configurations when $P_{p}=3 \mathrm{~dB}, P_{s}=5 \mathrm{~dB}$, and $d=0.4$.

packet is successfully transmitted from the PU to the PR through the primary link directly. The probability of the event $\mathrm{A}$ is given by

$$
\operatorname{Pr}(\mathrm{A})=\frac{\operatorname{Pr}\left(\bar{O}_{P, R}^{t}\right)}{\operatorname{Pr}\left(\bar{O}_{P, R}^{t}\right)+\operatorname{Pr}\left(O_{P, R}^{t}\right) \operatorname{Pr}\left(\bar{O}_{P, S}^{t}\right)}=\frac{\mu_{P N}^{\max }}{\mu_{P C}^{\max }} .
$$

To this end, the average end-to-end delay for the packet of the queue $Q_{p}$ in the proposed cooperative scheme is given by

$$
\bar{T}_{P C}=\bar{T}_{P} \operatorname{Pr}(\mathrm{A})+\left(\bar{T}_{P}+\bar{T}_{S P}\right) \operatorname{Pr}(\overline{\mathrm{A}}) .
$$

The closed-form expressions for the average delay of $\bar{T}_{P}$ and $\bar{T}_{S P}$ can be derived by following the similar process

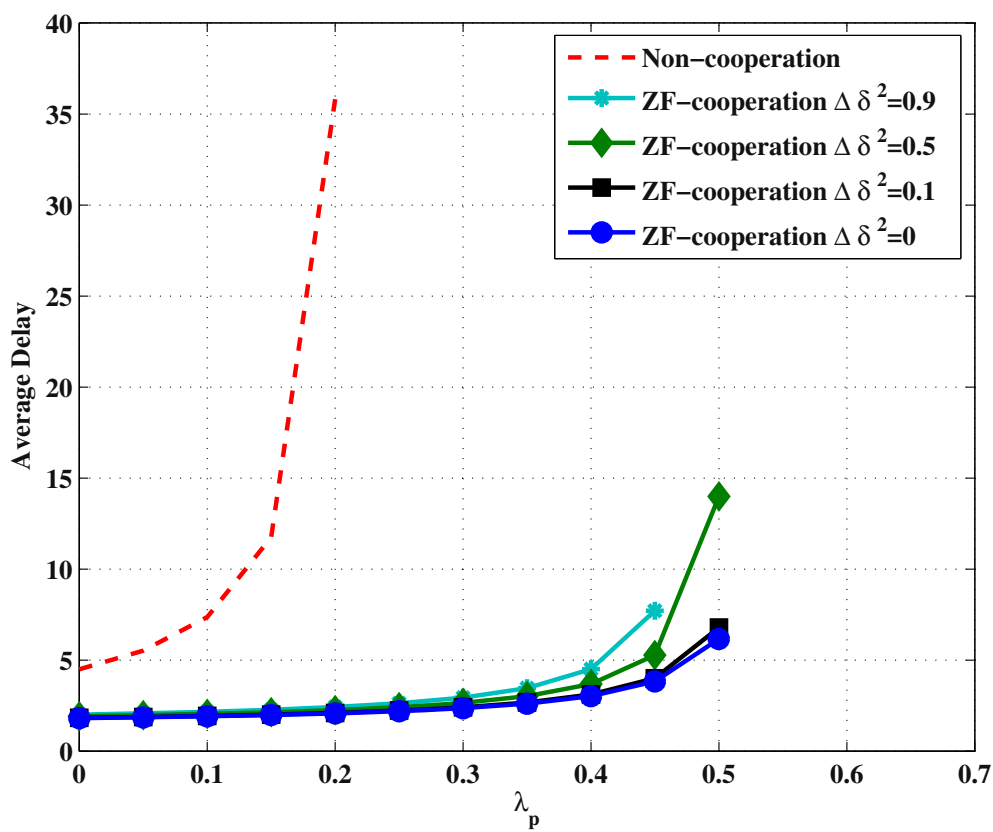

Figure 10 Average queue delay for PU versus $\lambda_{p}$ under imperfect CSI when $M=3, P_{p}=3 \mathrm{~dB}, P_{s}=5 \mathrm{~dB}$ and $d=0.4$. 
in the non-cooperative access scheme. This completes the proof of Theorem 4.

\subsection{Results and discussions}

Here we compare the average end-to-end PU's queue delay of the proposed ZF-cooperation scheme with that of the non-cooperation scheme. We now consider the following two cases of interest in the simulations. Case 1: SU owning perfect CSI and Case 2: SU owning imperfect CSI.

Figures 9 and 10 illustrate that the delay performance of the proposed cooperative access scheme versus the PU's arrival rate $\lambda_{p}$ under perfect CSI and imperfect CSI cases, respectively. As shown in Figure 9, the average end-toend delay performance of the proposed ZF-cooperation scheme outperforms that of the non-cooperation scheme. Moreover, the packet of the PU's queue suffers severe delay with the increasing of the PU's arrival rate. However, increasing the number of antennas can significantly improve the average end-to-end delay performance of the proposed ZF-cooperation scheme due to more diversity gain. In Figure 10, we investigate the impact of the imperfect CSI on the average end-to-end delay performance for the PU with different antenna configurations. It is observed that the average delay of the PU is almost not affected by the imperfect CSI at low arrival rate and can be improved by increasing the number of antennas. In addition, the proposed cooperative access scheme still outperforms the non-cooperative access scheme under the imperfect CSI, which demonstrates that the proposed cooperative access scheme keeps robust against the imperfect CSI at the SU.

\section{Conclusions}

In this article, we have dealt with the protocol design in cognitive cooperative networks with multiple antennas. To simultaneously transmit the PU's data and its own data based on the superposition coding such that they were received without interference at the $P R$ and $S R$ respectively, two weight vectors were designed at the SU based on zero-forcing algorithm. Specifically, we analyzed the maximum stable throughput and the average end-to-end delay performance of the proposed cooperative access scheme based on zero-forcing algorithm and compared with that of the existing cooperative access scheme based on the DPC. Simulation results demonstrated that the proposed cooperative access scheme achieved a better performance than the existing cooperative access scheme by using the DPC. In addition, the cooperative stable throughput gains for the PU and the SU were defined to study the effect of antennas on the performance of cognitive cooperative networks, and the upper bounds for the PU and the SU were also derived, respectively. Through analysis and simulations, we found that the upper bound of cooperative gain could be obtained by increasing the transmit power of the SU or the number of antennas. Furthermore, the impact of imperfect CSI on the performance of the proposed cooperative access scheme was investigated from the practical view and simulation results showed that the proposed cooperative access scheme kept robust against the imperfect CSI at the SU.

\section{Endnotes}

${ }^{\text {a }}$ The system model, corresponding to a practical scenario in which a secondary base station is serving one $\mathrm{SU}$, is also considered in [15].

${ }^{\mathrm{b}}$ Assuming that the packet ACK or NACK feedback is no error due to the fact that the short length of ACK/NACK packets can be coded at very low rate in the feedback channel [11-13].

${ }^{\mathrm{c}}$ In practice, the CSI between the SU and the SR can be obtained through the classic channel estimation and feedback mechanisms as in [30]. While the CSI about $\mathbf{g}_{0}$ and $\mathbf{f}_{1}$ can be obtained at the SU by direct estimating pilot signals from the primary system or by using the band manager which can exchange the channel information between the PU and the SU [31].

\section{Competing interests}

The authors declare that they have no competing interests.

\section{Acknowledgements}

This work was supported by the National Basic Research Program of China under Grant No. 2009CB320400, the National Science Foundation of China under Grant Nos. 60932002, 61072044 and 61172062, and in part by the Jiangsu Province Natural Science Foundation under Grant No. BK2011116.

Received: 28 August 2012 Accepted: 20 February 2013 Published: 14 March 2013

\section{References}

1. Federal Communications Commission, Spectrum policy task force (Nov 2002). Rep. ET Docket no 02-135

2. A Goldsmith, S Jafar, Breaking spectrum gridlock with cognitive radios: an information theoretic perspective. Proc IEEE. 97(5), 894-914 (2009)

3. MO Hasna, MS Alouini, End-to-end performance of transmission systems with relays over Rayleigh-fading channels. IEEE Trans. Wirel. Commun. 2(6), 1126-1131 (2003)

4. G Ganesan, Y Li, Cooperative spectrum sensing in cognitive radio, part I: two user networks. IEEE Trans. Wirel. Commun. 6(6), 2204-2213 (2007)

5. HT Yucek, A Arslan, survey of spectrum sensing algorithms for cognitive radio applications. IEEE Commun. Surv. Tutor. 11(1), 116-130 (2009)

6. TCui, F Gao, A Nallanathan, Optimization of cooperative spectrum sensing in cognitive radio. IEEE Trans. Veh. Technol. 60(4), 1578-1589 (2011)

7. G Taricco, Optimization of linear cooperative spectrum sensing for cognitive radio networks. IEEE J. Sel. Areas Commun. 5(1), 77-86 (2011)

8. Y Zou, Y-D Yao, B Zheng, Cognitive transmissions with multiple relays in cognitive radio networks. IEEE Trans. Wirel. Commun. 10(2), 648-659 (2011)

9. Y Han, SH Ting, A Pandharipande, Cooperative spectrum sharing protocol with secondary user selection. IEEE Trans. Wirel. Commun. 9(9), 2924-2923 (2011)

10. Q Li, SH Ting, A Pandharipande, Y Han, Cognitive spectrum sharing with two-way relaying systems. IEEE Trans. Veh. Technol. 60(3), 1233-1240 (2011)

11. O Simeone, Y Bar-Ness, U Spagnolini, Stable throughput of cognitive radios with and without relaying capability. IEEE Trans. Commun. 55(12) 2351-2360 (2007) 
12. AK Sadek, KJ Ray Liu, A Ephremides, Cognitive multiple access via cooperation: protocol design and performance analysis. IEEE Trans. Inf. Theory. 53(10), 3677-3696 (2007)

13. I Krikidis, JN Laneman, JS Thompson, S McLaughlin, Protocol design and throughput analysis for multi-user cognitive cooperative systems. IEEE Trans. Wirel. Commun. 8(9), 4740-4751 (2009)

14. X Bao, P Martins, T Song, L Shen, Stable throughput and delay performance in cognitive cooperative systems. IET Commun. 5(2), 190-198 (2010)

15. R Manna, RHY Louie, Y Li, B Vucetic, Cooperative spectrum sharing in cognitive radio networks with multiple antennas. IEEE Trans. Signal Process. 59(11), 5509-5522 (2011)

16. V Bohara, SH Ting, Y Han, A Pandharipande, in Proc. 5th Int. Conf. on Cognitive Radio Oriented Wireless Networks and Commun. (CROWNCOM). Interference free overlay cognitive radio network based on cooperative space time coding (Cannes, France, (June 2010)), pp. 1-5

17. Q Wu, Y Huang, J Wang, Y Cheng, Effective capacity of cognitive radio systems with GSC diversity under imperfect channel knowledge. IEEE Commun. Lett. 16(11), 1792-1795 (2012)

18. D Li, Performance analysis of MRC diversity for cognitive radio systems. IEEE Trans. Veh. Technol. 61(2), 849-853 (2012)

19. HL Van Trees, Optimum Array Processing. (Wiley, New York, 2002)

20. P Setoodeth, S Haykin, Robust transmit power control for cognitive radio. IEEE Proc. 97(5), 915-939 (2009)

21. IS Gradshteyn, IM Ryzhik, Table of Integrals, Series, and Products, 7th edn. (Academic, New York, 2007)

22. R Rao, A Ephremides, On the stability of interacting queues in a multi-access system. IEEE Trans. Inf. Theory. 34(5), 918-930 (1988)

23. RM Loynes, The stability of a queue with non-independent inter-arrival and service times. Math. Proc. Camb. Phil. Soc. 58(3), 497-520 (1962)

24. L Kleinrock, Queueing Systems Volume l: Theory. (Wiley, Canada, 1975)

25. J Liu, W Chen, Z Cao, YJ (Angela) Zhang, A distributed beamforming approach for enhanced opportunistic spectrum access in cognitive radios, (Hawaii, USA, (Nov 2009)), pp. 1-6

26. A Basilevsky, Applied Matrix Algebra in the statistical Science. (Wiley North-Holland, New York, 1983)

27. DS Michalopoulos, HA Suraweera, GK Karagiannidis, R Schober, Amplify-and-forward relay selection with outdated channel state information, (Miami, USA, (Dec 2010)), pp. 1-6

28. JL Vicario, A Bel, JA Lopez-Salcedo, G Seco, Opportunistic relay selection with outadated CSI: outage probability and diversity analysis. IEEE Trans. Wirel. Commun. 8(6), 2872-2876 (2009)

29. Y Ma, D Zhang, A Leith, Z Wang, Error performance of transmit beamforming with delayed and limited feedback. IEEE Trans. Wirel. Commun. 8(3), 1164-1170 (2009)

30. B L Tong, M Sadler, Dong, Pilot-assisted wireless transmissions: general model, design criteria, and signal processing. IEEE Trans. Signal Process. 21(6), 12-25 (2004)

31. A Ghasemi, ES Sousa, Fundamental limits of spectrum-sharing in fading environment. IEEE Trans. Wirel. Commun. 6(2), 649-658 (2007)

doi:10.1186/1687-1499-2013-70

Cite this article as: Huang et al:: Protocol design and performance analysis for cognitive cooperative networks with multiple antennas. EURASIP Journal on Wireless Communications and Networking 2013 2013:70.

\section{Submit your manuscript to a SpringerOpen ${ }^{\circ}$ journal and benefit from:}

- Convenient online submission

- Rigorous peer review

- Immediate publication on acceptance

- Open access: articles freely available online

- High visibility within the field

- Retaining the copyright to your article

Submit your next manuscript at $\boldsymbol{\wedge}$ springeropen.com 